\title{
Parallel polarization state generation and measurement with a single metasurface (Conference Presentation) (Withdrawal Notice)
}

Noah Rubin, Aun Zaidi, Ruo Ping Li, Michael Juhl, Jan Phillipp Balthasar Mueller, et al.

Noah A. Rubin, Aun Zaidi, Ruo Ping Li, Michael Juhl, Jan Phillipp Balthasar Mueller, Robert C. Devlin, Kristjan Leosson, Federico Capasso, "Parallel polarization state generation and measurement with a single metasurface (Conference Presentation) (Withdrawal Notice)," Proc. SPIE 10539, Photonic Instrumentation Engineering V, 1053911 (4 April 2018); doi: 10.1117/12.2290416

SPIE. Event: SPIE OPTO, 2018, San Francisco, California, United States 


\title{
Parallel polarization state generation and measurement with a single metasurface (Conference Presentation Withdrawal Notice)
}

\author{
Noah A. Rubin \\ Harvard Univ. (United States) \\ Aun Zaidi \\ Massachusetts Institute of Technology (United States) \\ Ruo Ping Li \\ Univ. of Waterloo (Canada) \\ Michael Juhl , Jan Phillipp Balthasar Mueller \\ Univ. of Iceland (Iceland) \\ Robert C. Devlin \\ Harvard Univ. (United States) \\ Kristjan Leosson \\ Univ. of Iceland (Iceland) \\ Federico Capasso \\ John A. Paulson School of Engineering and Applied Sciences (United States)
}

Proc. SPIE 10539, 1053911 (2018)

Online Publication Date: 14 March 2018

Withdrawn from Publication: 22 March 2018

Conference Date: 30 January-1 February 2018

Conference Location: San Francisco, California, United States

Conference Title: Photonic Instrumentation Engineering V

Conference Chairs: Yakov G. Soskind

Publisher's Note: This conference presentation recording, originally published on 14 March 2018, was withdrawn per author request.

ID 5751530557001 